\title{
THE CCT REVOLUTION
}

\author{
F. J. Schulte
}

Revolutions are rare, in particular those not accompanied by shedding or drawing - of blood. The invention of Cranial Computerized Tomography (CCT) by G. N. Hounsfield is in these days revolutionizing our diagnostic approach in all clinical neurosciences.

In recent decades diagnostic procedures in medicine became more and more aggressive with sometimes quite remarkable complication rates including mortality. In this respect, neurodiagnostic methods were in the forefront and it was for this reason, that many neuropediatricians remained quite conservative, sometimes, unfortunately, at the cost of a missed brain tumor.

Cranial Computerized Tomography wiped out $90 \%$ of these ugly procedures. Brain scans with their exposure to inoculated radioactive material can almost be abandoned in neuropediatrics; if there really was any indication for CSF scintigrams in children, it has become difficult to find one now; there seems hardly to be a place left for pneumencephalograms, and angiograms can be reserved for a few selected cases mainly with vascular disorders and supratentorial tumors. The radiation exposure of CCT is comparable to a plain skull $\mathrm{x}$-ray. CCT competes favourably with gross neuropathology, and it even allows, to a certain extend, microneuropathology in situ: with sur- prising accuracy the malignancy of a posterior fossa tumor can be anticipated.

Some of us with a meticulous training in classical neurology aiming at the clinical diagnosis and exact localisation of a brain tumor may look at the CCT machine with hostile feelings. The progress is so remarkable, however, that no one dealing with neurological disorders can afford not to make CCT available for his patients.

This is the reason why we asked Dr. Kazner and his co-workers to write a Survey on this subject for our readers. Although it is the first one of its kind in pediatric literature, for many neuropediatric centers its content is already everyday practice. We thought, however, that the great wealth of Dr. Kazner's experience with the new method should be made available to everyone concerned with the child's brain. One can quite legitimately ask whether it was necessary to spend so many of the journal's pages for so many pictures, that this issue looks more like an atlas than like a sientific journal. Extraordinary events, however, like the introduction of CCT, require and justify extraordinary leeway for their pioneers and, moreover, reviewing Dr. Kazner's survey, we felt that we were going to save our subscribers another textbook. 